\title{
Multi-User Privacy: The Gray-Wyner System and Generalized Common Information
}

\author{
Ravi Tandon, Lalitha Sankar, H. Vincent Poor \\ Dept. of Electrical Engineering, \\ Princeton University, Princeton, NJ 08544. \\ Email: $\{$ rtandon,lalitha,poor $\} @$ princeton.edu
}

\begin{abstract}
The problem of preserving privacy when a multivariate source is required to be revealed partially to multiple users is modeled as a Gray-Wyner source coding problem with $K$ correlated sources at the encoder and $K$ decoders in which the $k^{t h}$ decoder, $k=1,2, \ldots, K$, losslessly reconstructs the $k^{t h}$ source via a common link of rate $R_{0}$ and a private link of rate $R_{k}$. The privacy requirement of keeping each decoder oblivious of all sources other than the one intended for it is introduced via an equivocation constraint $E_{k}$ at decoder $k$ such that the total equivocation summed over all decoders $E \geq \Delta$. The set of achievable $\left(\left\{R_{k}\right\}_{k=1}^{K}, R_{0}, \Delta\right)$ rates-equivocation $(K+2)$-tuples is completely characterized. Using this characterization, two different definitions of common information are presented and are shown to be equivalent.
\end{abstract}

\section{INTRODUCTION}

Information sources often need to be made accessible to multiple legitimate users simultaneously. However, not all data from the source should be accessible to all users. For example, a computer retailer may need to share the annual revenue of all computers sold with all the vendors but share vendor-specific sale information only with a particular vendor. Similarly, a business consulting firm may share general data about a specific market with all clients associated with that market but share client-specific strategies with only that client. In both cases, one can view sharing the public (shared by all) information via a common link and the private information via a dedicated link. Maximizing the rate over the common link allows the information source (retailer/consulting firm) to share the most allowed publicly with all clients; however, the privacy guarantee requires that no client has access to private data of the other clients. This paper develops an abstract model and a methodology to study this problem.

We model the problem of revealing partial source information to multiple users while keeping the data specific to each user private from other users as a Gray-Wyner source coding problem with $K$ correlated sources at the encoder and $K$ decoders in which the $k^{\text {th }}$ decoder, $k=1,2, \ldots, K$, losslessly reconstructs the $k^{t h}$ source via a common link of rate $R_{0}$ and a private link of rate $R_{k}$. We model the privacy requirement

The research was supported by the Air Force Office of Scientific Research MURI Grant FA-9550-09-1-0643, by the National Science Foundation Grants CNS-09-05398 and CCF-10-16671, and by a Fellowship from the Council on Science and Technology at Princeton University. of keeping each decoder oblivious of all sources other than the one intended for it via an equivocation constraint $E_{k}$ at decoder $k$ such that the total equivocation summed over all decoders $E \geq \Delta$.

Since privacy is an important aspect of this problem, it is natural to understand the maximal total equivocation that is achievable if the rate on the common link is set to the maximum achievable. On the other hand, imposing the constraint of maximal total equivocation may lead to perhaps a different limit on the maximal rate on the common link. In this paper, we show that both requirements, which are formally different definitions, yield the same formulation for the maximal rate on the common link. In keeping with the literature, this common rate is defined as the common information.

The common information of two correlated random variables has been defined independently by Wyner [1] and GácsKörner [2]. Wyner's definition of common information as applied to the two-user Gray-Wyner system (without privacy constraints) is the minimum rate on the common link such that the total information shared across all three links (one common and two private) does not exceed the source entropy. On the other hand, the Gács-Körner common information is the maximal entropy of a random variable that two noninteracting terminals can agree upon when one terminal has access to $X^{n}$ and the other to $Y^{n}$ where $X$ and $Y$ are correlated random variables. For two correlated variables $X$ and $Y$, the Wyner common information $C_{W}$, the Gács-Körner common information $C_{G K}$, and the mutual information of the two variables are related as $C_{G K} \leq I(X ; Y) \leq C_{W}$. Recently, the authors in [3] have generalized Wyner's definition of common information to $K$ variables, henceforth referred to as $B\left(X_{1}, X_{2}, \ldots, X_{K}\right)$ for $K$ correlated variables. While the definition naturally generalizes the two variable common information, the resulting common information does not satisfy a non-increasing property with $K$ as expected.

In this paper, we present two different definitions of common information: the first is the maximal rate on the common link for which the total equivocation is maximized, and the second is the maximal rate on the common link such that each user losslessly reconstructs its intended source at its entropy. 


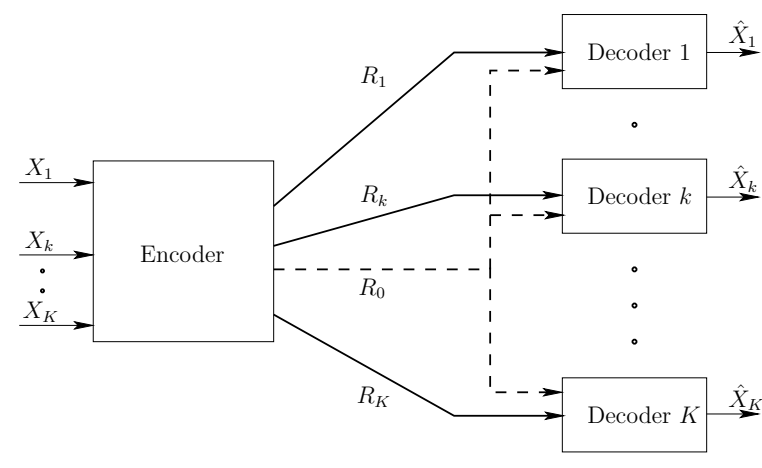

Fig. 1. The generalized Gray-Wyner source network.

We show that both definitions lead to the same formulation for common information $C\left(X_{1}, X_{2}, \ldots, X_{K}\right)$. We present many properties of $C\left(X_{1}, X_{2}, \ldots, X_{K}\right)$ and specifically show that $C\left(X_{1}, X_{2}, \ldots, X_{K}\right) \leq B\left(X_{1}, X_{2}, \ldots, X_{K}\right)$. To the best of our knowledge this is the first generalization of common information that preserves the non-increasing property and one whose form can be viewed as a natural generalization of the Gács-Körner common information to $K$ variables.

The paper is organized as follows. In Section III we present the system model. In Section III] we present the rate-equivocation region, develop a formulation for common information in two different ways, and present key properties. In Section IV] we compare our formulation with the $K$ variable generalization of Wyner's common information in [3] and illustrate with examples. We conclude in Section $\mathbf{V}$.

\section{SySTEM MODEL}

We consider the following source network. A centralized encoder observes $K$ discrete, memoryless correlated sources, $\left\{X_{k}^{n}\right\}_{k=1}^{K}$ and is interested in communicating source $X_{k}$ to decoder $k$ in a lossless manner. The resources available at the encoder comprise two types of noiseless rate-limited links. There are $K$ links of finite rate from the encoder to each of the $K$ decoders and there is a common link of finite rate to all decoders. Figure 1 shows the source broadcasting network in consideration.

An $\left(n,\left\{M_{k}\right\}_{k=1}^{K}, M_{0}\right)$ code for this model is defined by $(K+1)$ encoding functions described as

$$
\begin{aligned}
& f_{0}: \mathcal{X}_{1}^{n} \times \ldots \mathcal{X}_{K}^{n} \rightarrow\left\{1, \ldots, M_{0}\right\}, \\
& f_{k}: \mathcal{X}_{1}^{n} \times \ldots \mathcal{X}_{K}^{n} \rightarrow\left\{1, \ldots, M_{k}\right\}, \quad k=1, \ldots, K,
\end{aligned}
$$

and $K$ decoding functions,

$$
g_{k}:\left\{1, \ldots, M_{0}\right\} \times\left\{1, \ldots, M_{k}\right\} \rightarrow \mathcal{X}_{k}^{n}, \quad k=1, \ldots, K .
$$

We define the probability of error at decoder $k$ as

$$
P_{e, k}=\operatorname{Pr}\left(X_{k}^{n} \neq g_{k}\left(f_{0}\left(\bar{X}^{n}\right), f_{k}\left(\bar{X}^{n}\right)\right)\right),
$$

where $\bar{X}^{n} \triangleq\left\{X_{k}^{n}\right\}_{k=1}^{K}$. We define the equivocation at decoder $k$ as

$$
E_{k}=\frac{1}{n} H\left(\bar{X}^{n} \backslash X_{k}^{n} \mid f_{0}\left(\bar{X}^{n}\right), f_{k}\left(\bar{X}^{n}\right)\right),
$$

and the total equivocation as $E=\sum_{k=1}^{K} E_{k}$.

Remark 1: Informally, $E_{k}$ captures the average uncertainty, and hence privacy achievable, about the remaining $(K-1)$ unintended sources at decoder $k$.

An $\left(\left\{R_{k}\right\}_{k=1}^{K}, R_{0}, \Delta\right)$ rate-equivocation $(K+2)$-tuple is achievable for the source network if there exists an $\left(n,\left\{M_{k}\right\}_{k=1}^{K}, M_{0}\right)$ code such that,

$$
\begin{aligned}
M_{0} & \leq 2^{n R_{0}}, \\
M_{k} & \leq 2^{n R_{k}}, \quad k=1, \ldots, K \\
P_{e, k} & \leq \epsilon_{k}, \quad k=1, \ldots, K \\
E & \geq \Delta-\epsilon .
\end{aligned}
$$

We denote by $\mathcal{R}$ the region of all achievable $\left(\left\{R_{k}\right\}_{k=1}^{K}, R_{0}, \Delta\right)$ rate-equivocation $(K+2)$-tuples.

\section{MAIn CONTRIBUtions}

\section{A. Rate-Equivocation Region}

We state our first result in the following theorem. The proof is presented in the appendix.

Theorem 1: The region $\mathcal{R}$ of achievable rates-equivocation $(K+2)$-tuples for the source network shown in Figure 1 is the union of all $(k+2)$-tuples $\left(\left\{R_{k}\right\}_{k=1}^{K}, R_{0}, \Delta\right)$ that satisfy

$$
\begin{aligned}
R_{0} & \geq I\left(X_{1}, X_{2}, \ldots, X_{K} ; W\right), \\
R_{k} & \geq H\left(X_{k} \mid W\right), \quad k=1,2, \ldots, K, \\
\Delta & \leq \sum_{k=1}^{K} H\left(\bar{X} \mid W, X_{k}\right)
\end{aligned}
$$

where the union is over all auxiliary random variables $W$ arbitrarily correlated with $\left(X_{1}, X_{2}, \ldots, X_{K}\right)$, and where $\bar{X} \equiv$ $\left(X_{1}, X_{2}, \ldots, X_{K}\right)$.

Remark 2: The rate region $\mathcal{R}_{G-W}$ of the Gray-Wyner network without additional equivocation constraints is the region of $(K+1)$ rate tuples that satisfy (7) and (8).

\section{B. Common Information of $K$ Correlated Variables}

We now present two definitions for the common information of $K$ correlated random variables.

Definition 1: The common information of $K$ correlated random variables, $C_{1}$, is the maximal value of $R_{0}$, such that $\left(\left\{R_{k}\right\}_{k=1}^{K}, R_{0}, \Delta_{\max }\right) \in \mathcal{R}$, where

$$
\Delta_{\text {max }} \triangleq \sum_{k=1}^{K} H\left(\bar{X} \mid X_{k}\right) .
$$

Definition 2: The common information of $K$ correlated random variables, $C_{2}$, is the maximal value of $R_{0}$, such that $\left(\left\{H\left(X_{k}\right)-R_{0}\right\}_{k=1}^{K}, R_{0}\right) \in \mathcal{R}_{G-W}$.

We next state our second result.

Theorem 2: $C_{1}$ and $C_{2}$ are related as follows:

$$
C_{1}=C_{2}=\max _{W-X_{k}-\bar{X} \backslash X_{k}, k=1,2, \ldots, K} I\left(X_{1} X_{2} \ldots X_{K} ; W\right) .
$$

Proof: From Definition 1 the achievable equivocation $E$ must satisfy

$$
E \geq \Delta \max =\sum_{k=1}^{K} H\left(\bar{X} \mid X_{k}\right)
$$


On the other hand, any achievable $\left(\left\{R_{k}\right\}_{k=1}^{K}, R_{0}, E\right) \in \mathcal{R}$ also satisfies

$$
E \leq \sum_{k=1}^{K} H\left(\bar{X} \mid W, X_{k}\right)
$$

We therefore, have the following constraint:

$$
\sum_{k=1}^{K} H\left(\bar{X} \mid W, X_{k}\right) \geq \sum_{k=1}^{K} H\left(\bar{X} \mid X_{k}\right)
$$

which is equivalent to the following $K$ constraints:

$$
I\left(\bar{X} \backslash X_{k} ; W \mid X_{k}\right)=0, \quad k=1, \ldots, K .
$$

Therefore, from Definition 1, $C_{1}$ is equal to the maximal $R_{0}$ subject to (11), which implies that

$$
C_{1}=\max _{W-X_{k}-\bar{X} \backslash X_{k}, k=1, \ldots, K} I\left(X_{1}, \ldots, X_{K} ; W\right) .
$$

From Definition 2, $C_{2}$ is defined as the maximal $R_{0}$ such that $R_{k}+R_{0}=H\left(X_{k}\right)$, for $k=1, \ldots, K$, and $\left(\left\{R_{k}\right\}_{k=1}^{K}, R_{0}\right) \in \mathcal{R}_{G-W}$. We therefore have the following constraints for $k=1, \ldots, K$ :

$$
\begin{aligned}
H\left(X_{k}\right) & =R_{k}+R_{0} \\
& \geq H\left(X_{k} \mid W\right)+I\left(X_{1}, \ldots, X_{K} ; W\right) .
\end{aligned}
$$

These constraints are equivalent to

$$
I\left(\bar{X} \backslash X_{k} ; W \mid X_{k}\right)=0, \quad k=1, \ldots, K .
$$

Therefore, $C_{2}$ can be written as follows:

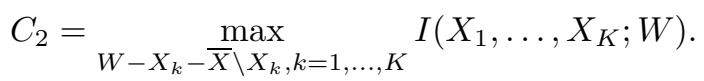

\section{Common Information: Properties}

We will now develop some properties of common information of $K$ correlated random variables defined in Theorem 2.

Proposition 1: The common information of $K$ random variables, $C\left(X_{1}, X_{2}, \ldots, X_{K}\right)$, is monotonically decreasing in $K$.

Proof: Consider an arbitrary $W$ satisfying the Markov chain relationship

$$
W-X_{k}-\bar{X} \backslash X_{k}, \quad k=1, \ldots, K .
$$

First consider the following sequence of inequalities:

$$
\begin{aligned}
& I\left(X_{1}, \ldots, X_{K-1}, X_{K} ; W\right) \\
& =I\left(X_{1}, \ldots, X_{K-1} ; W\right)+I\left(X_{K} ; W \mid X_{1}, \ldots, X_{K-1}\right) \\
& \leq I\left(X_{1}, \ldots, X_{K-1} ; W\right)+I\left(X_{2}, \ldots, X_{K} ; W \mid X_{1}\right) \\
& =I\left(X_{1}, \ldots, X_{K-1} ; W\right)
\end{aligned}
$$

where (17) follows from the Markov chain relationship $W$ $X_{1}-\left(X_{2}, \ldots, X_{K}\right)$. Now consider the following sequence of inequalities:

$$
\begin{aligned}
& C\left(X_{1}, \ldots, X_{K}\right) \\
& =\max _{W-X_{k}-\bar{X} \backslash X_{k}, \quad k=1, \ldots, K} I\left(X_{1}, \ldots, X_{K} ; W\right) \\
& \leq \quad{ }_{W-X_{k}-\bar{X} \backslash X_{k}, \quad k=1, \ldots, K} I\left(X_{1}, \ldots, X_{K-1} ; W\right) \\
& \leq \quad \max _{W-X_{k}-\bar{X} \backslash\left(X_{k}, X_{K}\right), \quad k=1, \ldots,(K-1)} I\left(X_{1}, \ldots, X_{K-1} ; W\right) \\
& =C\left(X_{1}, \ldots, X_{K-1}\right)
\end{aligned}
$$

where (19) follows from (17) and (20) follows from the fact that the Markov chain relationship $W-X_{k}-\bar{X} \backslash X_{k}$ implies the Markov chain relationship $W-X_{k}-\bar{X} \backslash\left(X_{k}, X_{K}\right)$. Since the random variable $X_{K}$ could be chosen arbitrarily from the set $\left(X_{1}, \ldots, X_{K}\right)$, (21) shows that the common information is monotonically decreasing in $K$.

Proposition 2: $C\left(X_{1}, X_{2}, \ldots, X_{K}\right)$ is upper bounded as

$$
C\left(X_{1}, X_{2}, \ldots, X_{K}\right) \leq \min _{i \neq j, i, j=1,2, \ldots, K} I\left(X_{i} ; X_{j}\right) .
$$

Proof: We consider an arbitrary $W$ satisfying (14), and upper bound the following mutual information:

$$
\begin{aligned}
I\left(X_{1}, \ldots, X_{K} ; W\right) & =I\left(X_{i} ; W\right)+I\left(\bar{X} \backslash X_{i} ; W \mid X_{i}\right) \\
& =I\left(X_{i} ; W\right) \\
& \leq I\left(X_{i} ; X_{j}, W\right) \\
& =I\left(X_{i} ; X_{j}\right)+I\left(X_{i} ; W \mid X_{j}\right) \\
& =I\left(X_{i} ; X_{j}\right)
\end{aligned}
$$

where (24) follows from the Markov chain condition $W-$ $X_{i}-\bar{X} \backslash X_{i}$, and (27) follows from the Markov chain condition $W-X_{j}-X_{i}$. The choice of $(i, j)$ was arbitrary, and therefore, the common information is upper bounded by the minimum of pairwise mutual information among all pairs, i.e.,

$$
C\left(X_{1}, \ldots, X_{K}\right) \leq \min _{i \neq j} I\left(X_{i} ; X_{j}\right) .
$$

\section{COMPARISON AND EXAMPLES}

In [1] Wyner defines the common information of two correlated random variables $\left(X_{1}, X_{2}\right)$ as

$$
B\left(X_{1}, X_{2}\right)=\inf _{X_{1} \rightarrow W \rightarrow X_{2}} I\left(X_{1}, X_{2} ; W\right) .
$$

One interpretation of this common information can be obtained from the Gray-Wyner source network. The common information $B\left(X_{1}, X_{2}\right)$ of two random variables is given as the smallest value of $R_{0}$ such that $\left(R_{1}, R_{2}, R_{0}\right) \in \mathcal{R}_{G-W}$ and $R_{0}+R_{1}+R_{2} \leq H\left(X_{1}, X_{2}\right)$. Recently, this notion of common information was generalized to $K$ correlated random variables in [3]. The common information, $B\left(X_{1}, \ldots, X_{K}\right)$, of $K$ correlated random variables, as defined in [3], is given by smallest value of $R_{0}$ such that $\left(\left\{R_{k}\right\}_{k=1}^{K}, R_{0}\right) \in \mathcal{R}_{G-W}$ and $R_{0}+\sum_{i=1}^{K} R_{k} \leq H\left(X_{1}, \ldots, X_{K}\right)$. The common information $B\left(X_{1}, \ldots, X_{K}\right)$ is given as

$$
B\left(X_{1}, \ldots, X_{K}\right)=\inf I\left(X_{1}, \ldots, X_{K} ; W\right)
$$


where the infimum is over all distributions $p\left(w, x_{1}, \ldots, x_{K}\right)$ that satisfy

$$
\begin{aligned}
\sum_{w \in \mathcal{W}} p\left(w, x_{1}, \ldots, x_{K}\right) & =p\left(x_{1}, \ldots, x_{K}\right) \\
p\left(x_{1}, \ldots, x_{K} \mid w\right) & =\prod_{k=1}^{K} p\left(x_{k} \mid w\right) .
\end{aligned}
$$

It was shown in [3] that $B\left(X_{1}, \ldots, X_{K}\right)$ is monotonically increasing in $K$. We believe that any intuitively satisfactory measure of common information should satisfy the property that the common information should decrease as the number of random variables increases. In Proposition 11 we showed that our measure of common information indeed satisfies this property.

We next prove a property of $B\left(X_{1}, \ldots, X_{K}\right)$ that helps us in comparing it with our common information $C\left(X_{1}, \ldots, X_{K}\right)$.

Proposition 3: $B\left(X_{1}, X_{2}, \ldots, X_{K}\right)$ is lower bounded as follows:

$$
\max _{i \neq j} I\left(X_{i} ; X_{j}\right) \leq B\left(X_{1}, X_{2}, \ldots, X_{K}\right) .
$$

Proof: To prove Proposition 3 consider an arbitrary $W$ satisfying the constraints (28)-(29) and the following sequence of inequalities:

$$
\begin{aligned}
I\left(X_{1}, \ldots, X_{K} ; W\right) & \geq I\left(X_{i} ; W\right) \\
& \geq I\left(X_{i} ; X_{j}\right)
\end{aligned}
$$

where (32) follows from the Markov chain relationship $X_{i}-$ $W-X_{j}$, and from the data processing inequality. In arriving at (32), the choice of $(i, j)$ was arbitrary, and therefore we can maximize over all pairs $(i, j)$ such that $i \neq j$ to get the best possible lower bound in this manner.

Using Propositions 2 and 3 , we have the following:

$$
\begin{aligned}
C\left(X_{1}, \ldots, X_{K}\right) & \leq \min _{i \neq j} I\left(X_{i} ; X_{j}\right) \\
& \leq \max _{i \neq j} I\left(X_{i} ; X_{j}\right) \leq B\left(X_{1}, \ldots, X_{K}\right) .
\end{aligned}
$$

We will now give two examples to illustrate the usefulness of our definition $C\left(X_{1}, \ldots, X_{K}\right)$ over $B\left(X_{1}, \ldots, X_{K}\right)$.

Example 1: Consider $K=3$ random variables $\left(X_{1}, X_{2}, X_{3}\right)$ such that $X_{1} \sim \operatorname{Ber}(1 / 2), X_{2}=X_{1} \oplus N$, where $N \sim \operatorname{Ber}(\delta)$ and $X_{3}$ is independent of $\left(X_{1}, X_{2}\right)$. Since $X_{3}$ is independent of $\left(X_{1}, X_{2}\right)$, these sources have nothing in common and we should expect the 'common information' to be zero. Note that for these sources, $\min _{i \neq j} I\left(X_{i} ; X_{j}\right)=0$, whereas $\max _{i \neq j} I\left(X_{i} ; X_{j}\right)=1-h(\delta)$. Therefore, from (33), we have

$$
0 \leq C\left(X_{1}, X_{2}, X_{3}\right) \leq 0 \leq 1-h(\delta) \leq B\left(X_{1}, X_{2}, X_{3}\right),
$$

which implies that $C\left(X_{1}, X_{2}, X_{3}\right)=0$, whereas $B\left(X_{1}, X_{2}, X_{3}\right)>0$ for any $\delta \in(0,1 / 2)$.

Example 2: Consider $K=3$ random variables $\left(X_{1}, X_{2}, X_{3}\right)$ such that $X_{1}=\left(X_{0}, X_{1 p}\right), X_{2}=\left(X_{0}, X_{2 p}\right)$ and $X_{3}=\left(X_{0}, X_{3 p}\right)$, where $\left(X_{0}, X_{1 p}, X_{2 p}, X_{3 p}\right)$ are all mutually independent. Since $X_{0}$ appears to be the only common part in all three sources, we should expect the 'common information' to be equal to the entropy of $X_{0}$. Note that for these sources, $\min _{i \neq j} I\left(X_{i} ; X_{j}\right)=\max _{i \neq j} I\left(X_{i} ; X_{j}\right)=H\left(X_{0}\right)$. Therefore, from (33), we have

$$
0 \leq C\left(X_{1}, X_{2}, X_{3}\right) \leq H\left(X_{0}\right) \leq B\left(X_{1}, X_{2}, X_{3}\right),
$$

It is straightforward to show that for these sources,

$$
C\left(X_{1}, X_{2}, X_{3}\right)=B\left(X_{1}, X_{2}, X_{3}\right)=H\left(X_{0}\right) \text {. }
$$

Inspired by the above example, we show the following interesting property that in some sense relates $C\left(X_{1}, \ldots, X_{K}\right)$ to $B\left(X_{1}, \ldots, X_{K}\right)$.

Proposition 4: For a set of sources $X_{1}, X_{2}, \ldots, X_{K}$ that satisfy

$$
\min _{i \neq j} I\left(X_{i} ; X_{j}\right)=\max _{i \neq j} I\left(X_{i} ; X_{j}\right),
$$

we have

$$
\begin{gathered}
C\left(X_{1}, X_{2}, \ldots, X_{K}\right)=\min _{i \neq j} I\left(X_{i} ; X_{j}\right) \\
\text { if } \quad B\left(X_{1}, X_{2}, \ldots, X_{K}\right)=\max _{i \neq j} I\left(X_{i} ; X_{j}\right) .
\end{gathered}
$$

Proof: The constraint (34) implies that the mutual information $I\left(X_{i} ; X_{j}\right)$ is the same for all $i, j \in\{1, \ldots, K\}$, $i \neq j$. Let us start with a $W^{*}$ that satisfies the infimization constraints for $B\left(X_{1}, \ldots, X_{K}\right)$ and yields

$$
\begin{aligned}
B\left(X_{1}, \ldots, X_{K}\right) & =\max _{i \neq j} I\left(X_{i} ; X_{j}\right) \\
& =I\left(X_{i_{0}} ; X_{j_{0}}\right),
\end{aligned}
$$

for some $i_{0} \neq j_{0}$. For this $W^{*}$, we have

$$
\begin{aligned}
I\left(X_{i_{0}} ; X_{j_{0}}\right) & =\max _{i \neq j} I\left(X_{i} ; X_{j}\right) \\
& =I\left(X_{1}, \ldots, X_{K} ; W^{*}\right) \\
& =I\left(X_{i_{0}} ; W^{*}\right)+I\left(\bar{X} \backslash X_{i_{0}} ; W^{*} \mid X_{i_{0}}\right) \\
& \geq I\left(X_{i_{0}} ; X_{j_{0}}\right)+I\left(\bar{X} \backslash X_{i_{0}} ; W^{*} \mid X_{i_{0}}\right)
\end{aligned}
$$

where (42) follows from the fact that $W^{*}$ satisfies the Markov relationship $X_{i_{0}}-W^{*}-X_{j_{0}}$, for all $i_{0} \neq j_{0}$. In the derivation of (42), $i_{0}$ can be chosen arbitrarily due to (34). Therefore, (42) implies that this $W^{*}$ also satisfies

$$
I\left(\bar{X} \backslash X_{i} ; W^{*} \mid X_{i}\right)=0
$$

for all $i=1, \ldots, K$. This in turn implies that $W^{*}$ serves as a valid choice in the maximization for evaluation of $C\left(X_{1}, \ldots, X_{K}\right)$. Therefore, we obtain the following lower bound for $C\left(X_{1}, \ldots, X_{K}\right)$ :

$$
\begin{aligned}
C\left(X_{1}, \ldots, X_{K}\right) & =\max _{W-X_{k}-\bar{X} \backslash X_{k}, k=1, \ldots, K} I\left(X_{1}, \ldots, X_{K} ; W\right) \\
& \geq I\left(X_{1}, \ldots, X_{K} ; W^{*}\right) \\
& =\max _{i \neq j} I\left(X_{i} ; X_{j}\right) \\
& =\min _{i \neq j} I\left(X_{i} ; X_{j}\right) .
\end{aligned}
$$

Hence, from Proposition 11 it now follows that if $B\left(X_{1}, \ldots, X_{K}\right)=\max _{i \neq j} I\left(X_{i} ; X_{j}\right)$, then 
$C\left(X_{1}, \ldots, X_{K}\right)=\min _{i \neq j} I\left(X_{i} ; X_{j}\right)$. We remark here that a similar property has been shown for $K=2$ by Ahlswede and Körner in [4].

\section{CONCLUding REMARKS}

We have abstracted the problem of privacy in a setting where a source interacts with multiple users via the GrayWyner source coding problem with additional equivocation constraints at each user and a total equivocation constraint. In addition to developing the rate-equivocation region, we have introduced two definitions of common information of $K$ correlated variables and shown them both to have a form that can be viewed as a $K$-user generalization of the Gács-Körner common information (see also [4]).

\section{Appendix: Proof of Theorem 1}

The converse follows by minor modifications of the converse proof for the unconstrained Gray-Wyner problem [5] and is therefore omitted. We now outline the proof of achievability for Theorem 1 .

Codebook generation: Fix an input distribution $p\left(w \mid x_{1}, \ldots, x_{K}\right)$. Generate $2^{n I\left(X_{1}, \ldots, X_{K} ; W\right)}$ sequences according to the distribution $\prod_{t=1}^{n} p\left(w_{t}\right)$, and index these sequences as $w^{n}(i)$, for $i=1, \ldots, 2^{n I\left(X_{1}, \ldots, X_{K} ; W\right)}$. Independently and uniformly bin the $X_{k}^{n}$-sequences in $2^{n H\left(X_{k} \mid W\right)}$ bins, and index these bins as

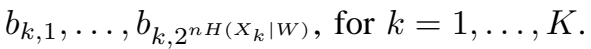

Encoding scheme: Upon observing the $\left(x_{1}^{n}, \ldots, x_{K}^{n}\right)$ sequences, the encoder searches for a $w^{n}$ sequence that is jointly typical with these sequences. Using standard arguments (as in [6]), it can be shown that the encoder can succeed in finding one such $w^{n}$ sequence. The encoder sends the index of the $w^{n}$ sequence on the public link, for which we require $R_{0} \geq I\left(X_{1}, \ldots, X_{K} ; W\right)$. It sends the bin index of the source sequence $x_{k}^{n}$ on the private link to decoder $k$, for which we require $R_{k} \geq H\left(X_{k} \mid W\right)$.

Decoding: At decoder $k$, the decoder looks for a unique $x^{n}$ in bin $b_{k}$ (received from the private link), that is jointly typical with the $w^{n}$ sequence received from the public link. It can be shown that decoder $k$ can reconstruct $X_{k}^{n}$ with a vanishingly small probability of error. We omit the probability of error calculation as it follows from the same arguments as in [5].

Equivocation: We show that this coding scheme yields the total equivocation stated in Theorem 1 . Let $J_{0}$ denote the encoder output for the public link and let $J_{k}$ denote the encoder output for the private link to decoder $k$, for $k=1, \ldots, K$. For $E_{k}$, we have the following sequence of inequalities:

$$
\begin{aligned}
E_{k}= & \frac{1}{n} H\left(X_{1}^{n}, \ldots, X_{k-1}^{n}, X_{k+1}^{n}, \ldots, X_{K}^{n} \mid J_{0}, J_{k}\right) \\
= & \frac{1}{n} H\left(\bar{X}^{n} \backslash X_{k}^{n} \mid J_{0}, J_{k}\right) \\
\geq & \frac{1}{n} H\left(\bar{X}^{n} \mid J_{0}, J_{k}\right)-\frac{1}{n} H\left(X_{k}^{n} \mid J_{0}, J_{k}\right) \\
\geq & \frac{1}{n} H\left(\bar{X}^{n} \mid J_{0}, J_{k}\right)-\epsilon_{k, n} \\
= & \frac{1}{n} H\left(\bar{X}^{n}, J_{0}, J_{k}\right)-\frac{1}{n} H\left(J_{0}, J_{k}\right)-\epsilon_{k, n} \\
\geq & \frac{1}{n} H\left(\bar{X}^{n}\right)-\frac{1}{n} H\left(J_{0}, J_{k}\right)-\epsilon_{k, n} \\
\geq & \frac{1}{n} H\left(\bar{X}^{n}\right)-\frac{1}{n} H\left(J_{0}\right)-\frac{1}{n} H\left(J_{k}\right)-\epsilon_{k, n} \\
\geq & H\left(X_{1}, \ldots, X_{K}\right)-I\left(X_{1}, \ldots, X_{K} ; W\right)-H\left(X_{k} \mid W\right) \\
& -\epsilon_{k, n} \\
= & H\left(X_{1}, \ldots, X_{K} \mid W, X_{k}\right)-\epsilon_{k, n} \\
= & H\left(\bar{X} \mid W, X_{k}\right)-\epsilon_{k, n}
\end{aligned}
$$

where (50) follows from Fano's inequality, and (54) follows from the facts that $H\left(J_{0}\right) \leq \log \left(\left|\mathcal{J}_{0}\right|\right)=$ $n I\left(X_{1}, \ldots, X_{K} ; W\right)$, and $H\left(J_{k}\right) \leq \log \left(\left|\mathcal{J}_{k}\right|\right)=$ $n H\left(X_{K} \mid W\right)$, for $k=1, \ldots, K$. Therefore, we have that

$$
E=\sum_{k=1}^{K} E_{k} \geq \sum_{k=1}^{K} H\left(\bar{X} \mid W, X_{k}\right)-\epsilon .
$$

Hence, this coding scheme yields an equivocation of $\Delta=$ $\sum_{k=1}^{K} H\left(\bar{X} \mid W, X_{k}\right)$.

\section{REFERENCES}

[1] A. D. Wyner, "The common information of two dependent random variables," IEEE Trans. Inform. Theory, vol. 21, no. 2, pp. 163-179, March 1975.

[2] P. Gács and J. Körner, "Common information is far less than mutual information," Problems of Control and Information Theory, vol. 2, pp. 149-162, 1973.

[3] W. Liu, G. Xu, and B. Chen, "The common information of $\mathrm{N}$ dependent random variables," in Proc. 48th Annual Allerton Conference on Communications, Control and Computing, Monticello, IL, September 2010.

[4] R. Ahlswede and J. Körner, "On common information and related characteristics of correlated information sources," in Lecture Notes in Computer Science. Berlin, Germany: Springer-Verlag, 2006, vol. 4123, pp. 664-677.

[5] R. M. Gray and A. D. Wyner, "Source coding for a simple network," Bell System Technical Journal, vol. 53, no. 9, pp. 1681-1721, November 1974.

[6] T. M. Cover and J. A. Thomas, Elements of Information Theory. New York: Wiley, 1991 